Psychology of Language and Communication 2013, Vol. 17, No. 3

VERSITAOPEN

DOI: $10.2478 /$ plc-2013-0018

FRANCISCO J. RODRÍGUEZ MUÑOZ

University of Almería

\title{
PILOT ASSESSMENT OF NONVERBAL PRAGMATIC ABILITY IN PEOPLE WITH ASPERGER SYNDROME
}

\begin{abstract}
The purpose of this study is to present a diagnostic tool to assess the nonverbal pragmatic behaviors of people with Asperger syndrome, with the intent to give an account of the severity of symptoms in the area of nonverbal interaction, as well as providing a profile of nonverbal behaviors that may be targeted for intervention. Through this communication profile, overall nonverbal ability is calculated in a group of 20 subjects with Asperger syndrome. The proposed scale also includes the measurement of the following nonverbal dimensions: (1) eye gaze, (2) facial expression, (3) body language and posture, (4) proxemics, (5) gestures, and (6) paralanguage. The results of this assessment suggest low nonverbal pragmatic ability in these subjects, show specific deficits in nonverbal communication, and capture variability in nonverbal behavior in individuals with AS.

Key words: Asperger syndrome, autism spectrum disorders, communication profile, nonverbal communication, pragmatic assessment, speech-language pathology
\end{abstract}

\section{Introduction}

Nobody can deny that nonverbal behavior, understood as a communication process, has a crucial role in every person's everyday interactions (Knapp, 1992; Poyatos, 1994; Ekman, 2004). On this matter, after analyzing the communication of feelings and attitudes, psychologist Mehrabian (1972) established that only $7 \%$ of all information is transmitted through words (verbal channel), while $38 \%$ refers to tone of voice and inflection (paralanguage), and the other $55 \%$ comes from body language (kinesics).

Throughout the present study, we highlight the transcendence of nonverbal communication in a group of subjects with Asperger syndrome (henceforth, AS),

Address for correspondence: Francisco J. Rodríguez Muñoz, Department of Philology, University of Almería, Ctra. Sacramento s/n, La Cañada de San Urbano, CP 04120 Almería, Spain. E-mail: frodriguez@ual.es 
as a part of social interaction deficits. In general, there are few references when it comes to assessing nonverbal abilities in individuals with communication pathologies and, more specifically, with autism spectrum disorders (henceforth, ASD). Because of this, it is necessary to develop evaluation tools that contribute to more efficient detection of nonverbal communication alterations. Therefore, the main objective of this research is the design of an evaluation profile of nonverbal behavior, intended for people with AS.

Authors like Tantam and Girgis (2009) agree that the fundamental difficulties presented by individuals with ASD are found in the area of social relations. Nonetheless, today there is still a discussion regarding the causes of this social disability. In their review, they propose that AS is basically a nonverbal communication disorder; that is, isolation and the resulting frustration of interpersonal contact are nothing more than the result of atypical nonverbal behavior.

Those who defend the possibility of reaching an early diagnosis, from two and three years of age, have directed clinical evaluation to the identification of nonverbal social behavior guidelines, although these behaviors are more perceptible at four or five years of age (Charman \& Baird, 2002).

People with AS perceive nonverbal inputs differently than neurotypicals and this is one of the underlying causes of their nonverbal communication impairments. Some researchers have concluded that their nonverbal skills do not improve with age (McGovern \& Sigman, 2005; Shattuck et al., 2007). These considerations are not ignored by diagnosis manuals, such as the DSM-IV-R (American Psychiatric Association, 2000) and the ICD-10 (World Health Organization, 1992), in which an alteration of certain "nonverbal behaviors, such as eye contact, facial expression, posture, and gesture" are pointed out as a manifestation of social interaction qualitative alterations.

Gillberg and Gillberg (1989) identify nonverbal communication difficulties in their symptom checklist. Even though they include "peculiar characteristics in rhythm, tone and prosody" in the section devoted to speaking and language problems, they take into consideration these characteristics directly related to nonverbal communication: (a) limited use of gestures; (b) clumsy body language; (c) limited facial expression; (d) inappropriate expression; and (e) peculiar or rigid gazing.

From our point of view, voice volume, rhythm and intonation are vocal components of speech that we call paralanguage and conceive, in the words of Poyatos (1994: 137), as nonverbal qualities and voice modifiers, and independent sounds and silences with which we support or contradict simultaneous or alternating verbal and kinetic structures.

According to Poyatos (1977), the triple basic structure of human communication behavior is composed of the linguistic level as well as the paralinguistic and kinetic systems. Specifically, kinetics studies body movements and facial expressions, i.e. psychomuscular movements and positions, conscious and unconscious, learned or somatogenic, of visual, audiovisual and tactile perception 
that, isolated or combined with linguistic and paralinguistic structures, as well as other somatic and objectual systems, possess communication value, intentional or not (Poyatos, 1994: 139). Therefore, the kinetic system, body language, body movements, facial expressions, even eye gaze and posture, are categories linked to one another, and consequently are interdependent (Nascimiento, 2005).

Body language, one of the most explored nonverbal categories in specialized literature, is defined as visible actions serving to produce an explicit communication act that is generally directed to an interlocutor, and with which speakers transmit their intentions (Kendon, 1984). Along with kinetics and paralanguage, the proxemic behaviors of speakers have to be included in a nonverbal communication outline (Hall, 1963). This includes the use of space during interactions, as well as the distance or interpersonal zone that mediates between interlocutors and ongoing physical contact between them while they communicate (RodríguezMuñoz, 2012: 24).

Following Cestero (2006), kinetics and paralanguage are primary systems because they are directly related to any human communication act, they work simultaneously with the verbal system when the speaker produces any utterance. Proxemics and chronemics - which is related to structure and use of time - are secondary systems, as they add social or cultural information, and thus modify or reinforce the meaning of primary system elements.

Given that people with AS present meaningful nonverbal communication deficits, correct detection of these will help to accurately diagnose and treat the disorder (Rodríguez-Muñoz, 2009; Rodríguez-Muñoz, 2011; Rodríguez-Muñoz, 2013; Rodríguez-Muñoz \& Ridao, 2011). Up until now, little interest has been shown in the assessment of these nonverbal components of communication. This is why professionals tend to find themselves without proper evaluation tools that would allow them to identify these kinds of alterations in patients and intervene effectively in their treatment.

According to the clinical pragmatic benchmark, such as the Quick Protocol for Pragmatic Assessment (QPPA) (Gallardo, 2007, 2009a, 2010), and having reviewed other primary references of communication evaluation (Prutting \& Kittchner, 1987; Bishop, 1998), this research proposes a new scale that will allow to assess nonverbal pragmatic behaviors in people with AS. To achieve this, six nonverbal basic categories will be taken into account: (a) eye gaze; (b) facial expression; (c) body language and posture; (d) proxemics; (e) gestures; and (f) paralanguage.

\section{Eye gaze}

There are many studies that support the assumption that the most frequent nonverbal behavior related to eye gaze that individuals with AS exhibit is averting their gaze (Klin et al., 2002; Dalton et al., 2005; Neumann et al., 2006; Hernández et al., 2009). However, there are exceptions where this rule is contrasted with its opposite, gazing. While avoiding direct eye contact can be a nonverbal character- 
istic symptom in these subjects, gazing can be an artificial and acquired behavior that can generate a feeling of intimidation in the interlocutor, even when this is not the speaker's intention. Additionally, avoiding eye contact can be linked to another social perception such as lack of attention, and it does not necessarily respond to this behavior either.

In relation to eye gaze, the profile recognizes two nonverbal items: (1) Eye contact and (2) Type of eye gaze. With the former, the evaluator considers whether the patient with AS can maintain eye contact with the interlocutor, while the latter is used to assess the naturalness of the gaze.

\section{Facial expression}

Many studies, usually with theory of mind as the framework (Baron-Cohen, Leslie, \& Frith, 1985), have paid attention to the difficulties individuals with AS have when it comes to interpreting emotions from facial expressions (Braverman et al., 1989; Grossman et al., 2000). Nonetheless, the interest given to limited, rigid or inappropriate facial expression has not been the same, even though it is recognized by diagnostic manuals.

The evaluation profile incorporates two communication elements for this category: (3) Type offacial expression, marked as positive when it is of complementary character, and not a substitution for verbal expression; and (4) Appropriateness of facial expressions, which is related to the convenience degree between facial expression, the content of linguistic messages, context, as well as the emotional state of the participant.

\section{Body language and posture}

Even though body language perception by individuals with AS has already been studied (Reed et al., 2007; Doody \& Bull, 2011; Rodríguez-Muñoz, 2012), there have been few attempts to evaluate this feature that diagnostic manuals and classic studies like those of Gillberg and Gillberg (1989) or Szatmari, Brenner and Nagy (1989) emphasize. It is also a clear indicator of the difficulties of social integration that this population presents (Attwood, 2000).

According to these criteria, item (5) Posture appropriateness to the media and the interlocutor is marked as positive when body language is successful during communication exchange, and it transmits a receptive attitude from the participant.

\section{Proxemics}

Proxemics is a fundamental component of nonverbal human communication. Item (6) Proxemic appropriateness is related to the use and processing of the physical space around the individuals. Specifically, this item is marked as positive when physical contact is established with the interlocutor and the distances between the participants during interactions are always those expected according to contextual conditions. 


\section{Gestures}

Even though gestures are a nonverbal category that can have many approaches and classifications, our profile focuses on three aspects: (7) Type of gesture, which evaluates the naturalness of gestures during interactions, besides its complementary character, and not as a substitution for verbal expression; (8) Amount of gestures, which takes into account the number of gestures speakers make while exchanging information; and (9) Appropriateness of gestures, which relies on the capacity of adaptation of this nonverbal channel to linguistic messages, context and the emotional state of the participants.

Also, an item is added as (10) Involuntary gestures (tics). It is marked as positive when the speaker does not produce any involuntary or convulsing movements during communicative exchange. This is one of the most frequent complications that children and adults with AS show. They can even present comorbid pathologies defined by stereotyped movements, like Gilles de la Tourette syndrome (Ringman \& Jankovic, 2000).

\section{Paralanguage}

Since the term paralanguage is often used to refer to nonverbal elements of speech, five items are selected for the evaluation of this paraverbal language dimension: (11) Voice volume, marked as positive when it is enough for intelligibility, without being excessively loud or low; (12) Rhythm and inflection, marked as positive when rhythmic patterns are regular in elocution, without any sudden variations in acceleration or deceleration; (13) Breathing, which takes into account that the participant has to regulate his or her breathing properly while producing verbal messages.

Irregularities in the quality of the components involved in speech production and phonation suggested the inclusion of (14) Oral alterations in our scale; other pragmatic protocols also pay attention to these problems that accompany the spoken word (cf. Gallardo, 2007, 2009a, 2009b, 2010; Gallardo \& Moreno, 2005; Gallardo \& Sanmartín, 2005; Garayzábal, 2005; Gallardo \& Moreno, 2011; Gallardo, Moreno, \& Pablo, 2011; Rodríguez-Muñoz, 2009, 2013). This item is evaluated positively when the speaker does not show the main articulation problems or disorders associated with oral language. It is evaluated negatively when a subject with dyslalia has difficulties when it comes to articulating sounds with the tongue; those affected by dysphemia or stuttering and, because of this, producing repetitions or audibly and involuntarily prolonging syllables or words, disrupting the fluidity of oral expression; or those presenting with echolalia, which is the involuntary repetition of words or sentences spoken by the interlocutor, in his presence as echoes. This last disturbance, according to Sigman and Capps (2000), develops in approximately $80 \%$ of verbal autistics. 
Finally, the profile incorporates item (15) Voice imitation, a typical Asperger trait, because even if imitative behaviors are not restricted to voice properties alone, many subjects with AS are great imitators (Attwood, 2006). To sum up, the detection of kinetic, paralinguistic and proxemic deficits will be of great help in learning more and for qualifying diagnostic nonverbal criteria so often related to AS by clinical manuals and specialists.

\section{Method}

The first step of this and other studies (see Roth and Spekman, 1984) was to describe the organizational framework for the assessment of pragmatic abilities - in this case, nonverbal behaviors in people with AS. To this aim, it was necessary to establish pertinent communicative items and present parameters in coordination with a review of literature. Based on such a review of the dimensions of deficit in nonverbal behavior exhibited by people with AS, a tool was designed to exclusively assess nonverbal pragmatic ability, as shown in Table 1.

This one-group pilot study - without a control group - employs a preexperimental design; therefore, it was conducted in order to gain first insights into the viability of the proposed nonverbal communication assessment scale. Although it would have been desirable to include comparison groups, it does not mean that the present evaluation is less rigorous or the results less significant. We cannot forget, either, that an important part of test construction is that the instrument should undergo item analysis (see Clapham, 2013).

According to other assessment materials in clinical linguistics, our profile follows the principles and instructions that its main antecedent, the Quick Protocol for Pragmatic Assessment (QPPA) (Gallardo, 2007, 2009a, 2010; RodríguezMuñoz, 2013), applies to different pathological populations - aphasia (Gallardo \& Moreno, 2005; Gallardo \& Sanmartín, 2005; Hernández, Serra y Veyrat, 2007), attention deficit hyperactivity disorder (ADHD) (Gallardo, 2009b), Williams syndrome (Garayzábal, 2005; Gallardo, 2010), right hemisphere brain damage (RHD) (Gallardo \& Moreno, 2011; Gallardo, Moreno \& Pablo, 2011), etc., where control groups are not used and ideal performance of communicative behaviors is ascribed to neurotypical groups.

\section{Participants}

Twenty individuals with AS participated in this pilot research study. The clinical diagnosis of AS was validated by psychologists who assisted in data collection. We also used an independent tool to verify the presence of an ASD diagnosis, i.e. the Social Communication Questionnaire (SCQ) (Rutter, Bailey, \& Lord, 2003).

Personal interviews were scheduled for each participant and took place in Asturias and Valencia, thanks to the collaboration of the Asperger Asturias and Asperger Valencia associations. With their parents' consent, the participants were 
Table 1. Profile for the assessment of nonverbal behaviors in people with AS

\section{Nonverbal behaviors are consistent with these statements}

\section{Eye gaze}

1. Eye contact

The subject keeps expected eye contact with the interlocutor

2. Type of eye gaze

The gaze is natural (for example, it is not extremely fixed or shifty)

\section{Facial expression}

3. Type of facial expression

Facial expressions are sufficient: they complement verbal expression and do not substitute it

4. Appropriateness of facial expressions

Facial expressions adapt to the content of the linguistic messages, context, and are coherent with the subject's emotional state

\section{Body language and posture}

5. Posture appropriateness

Body language and posture is appropriate during the exchange (for example, it is not excessively rigid or hyperkinetic and it transmits a receptive attitude)

\section{Proxemics}

6. Proxemic appropriateness

Physical contact with the interlocutor and the distances between them during the interaction are appropriate according to context

\section{Gestures}

7. Type of gestures

Gestures are natural; they complement verbal expression and do not substitute it

8. Amount of gestures

Amount of gestures made by the subject are enough, not limited or excessive

9. Appropriateness of gestures

Gestures are always adjusted to the linguistic messages they accompany, to the context and are coherent with the subject's emotional state

10. Involuntary gestures (tics)

Involuntary and convulsive gestures are null during verbal interaction

\section{Paralanguage}

11. Voice volume

The tone used during interaction is enough for intelligibility, without being excessively loud or low

12. Rhythm and inflection

The rhythm of speech is moderated, without any sudden variations in acceleration or deceleration; and it is not monotonous

13. Breathing

The subject regulates breathing appropriately while producing verbal emissions

14. Oral alterations

The subject can articulate utterances correctly, without any oral difficulties (for example, dyslalia, dysphemia or echolalia)

15. Voice imitation

Speech has no tonal imitation of other voices (for example, when reproducing in direct style a fragment produced by another speaker) 
videotaped for later analysis. The interactions came to 149.3 minutes and they were transcribed in ordinary orthography using the basic notation conventions of conversation analysis, due to the pragmatic nature of other analyses derived from a larger study that included not only nonverbal but also verbal communication deficits in people with AS (Rodríguez-Muñoz, 2013).

The ages of the participants, as shown in Table 2, ranged between 6 and 15 years, with an average of 11.15 years. Nineteen of them were male and there was only one recording of a female. Also, at the moment of data collection, they were not undergoing any pharmacological treatment.

\section{Assessment procedure}

According to the objectives of this study, efforts were made to establish a pattern of nonverbal communication behavior for the 20 individuals forming the

Table 2. Information about participants with AS

\begin{tabular}{|c|c|c|c|c|}
\hline Identifier & Gender & Age & Language & Ethnic origins \\
\hline AGC & M & 9 & Spanish (northern variety) & Avilés, Asturias (Spain) \\
\hline $\mathrm{DCF}$ & M & 11 & Spanish (northern variety) & Oviedo, Asturias (Spain) \\
\hline APC & M & 9 & Spanish (northern variety) & Oviedo, Asturias (Spain) \\
\hline BFG & M & 13 & Spanish (northern variety) & Oviedo, Asturias (Spain) \\
\hline ACR & M & 6 & Spanish (northern variety) & Cangas de Onís, Asturias (Spain) \\
\hline $\mathrm{MBF}$ & M & 15 & Spanish (northern variety) & Oviedo, Asturias (Spain) \\
\hline IGL & M & 12 & Spanish (northern variety) & Avilés, Asturias (Spain) \\
\hline DLC & M & 14 & Spanish (northern variety) & Gijón, Asturias (Spain) \\
\hline DGM & M & 13 & Spanish (northern variety) & Oviedo, Asturias (Spain) \\
\hline VGC & M & 11 & Spanish (northern variety) & Avilés, Asturias (Spain) \\
\hline JIS & M & 12 & Spanish (northern variety) & Oviedo, Asturias (Spain) \\
\hline AFM & M & 12 & Spanish (northern variety) & Oviedo, Asturias (Spain) \\
\hline FMS & M & 12 & Spanish (northern variety) & Mieres, Asturias (Spain) \\
\hline SAA & M & 10 & Spanish (northern variety) & Mieres, Asturias (Spain) \\
\hline JARC & M & 10 & Spanish (northern variety) & Valencia, Valencian Community (Spain) \\
\hline JORC & M & 8 & Spanish (northern variety) & Valencia, Valencian Community (Spain) \\
\hline DMF & M & 11 & Spanish (northern variety) & Valencia, Valencian Community (Spain) \\
\hline PGM & M & 11 & Spanish (northern variety) & Valencia, Valencian Community (Spain) \\
\hline CMR & $\mathrm{F}$ & 10 & Spanish (northern variety) & Valencia, Valencian Community (Spain) \\
\hline AFB & M & 14 & Spanish (northern variety) & Valencia, Valencian Community (Spain) \\
\hline
\end{tabular}


sample. To achieve this, an individual evaluation with each subject took place beforehand. As with Niemi et al. (2010), our observations were based on dyadic therapist-child conversations and on picture description tasks. The same pictures were shown to each of the AS subjects and all of them received the same instructions. Sessions lasted 7.47 minutes on average and were managed by a team of four psychologists known by the participants. This way, 15 communication items were applied, distributed into six nonverbal categories.

From the 20 subjects that were assessed, an average of scores was calculated in two different directions: (a) in general, nonverbal pragmatic ability was estimated (NPA); and (b) particularly, the percentage of achievement that each of the nonverbal communicative items had in total was taken into account, with all the participants.

For scoring, the profile only takes into account the positive annotations (yes/ observed), which are divided by the total of evaluated items (yes + no), not taking into consideration those that could not be examined (not observed or absent). Once the count was made, the value was multiplied by 100 , which results in the percentage of NPA for each of the subjects with communication pathology. Thus, an ideal NPA would be equal to $100 \%$ of all items marked positively, while lower values would indicate nonverbal deficits in people with AS.

Two independent evaluators assigned scores to the observations and participants in this study; we achieved an average $87 \%$ agreement between the two and found that most of the differences were due to divergent interpretations of the items rather than the observations of participants' nonverbal communicative behavior.

\section{Results and discussion}

First of all, this section shows the results relative to the NPA of each of the participants, and also presents the average of that indicator applied to the 20 subjects that make up the pathological sample. Later on, the scores that the participants obtained in each of the nonverbal items that are part of the scale are provided.

\section{Nonverbal pragmatic ability}

Table 3 presents the percentages relative to the NPA of each participant. According to the evaluation, the NPA average is $57.05 \%$ for the whole group. Consequently, this pilot assessment suggests significant altered or disordered nonverbal behaviors in people with AS.

\section{Nonverbal communication dimensions}

This section discusses nonverbal dimensions and their related items in order to determine the weakest points shared by the participants. 
Table 3. Scores for each participant (NPA)

\begin{tabular}{lrlr}
\hline Participant & \multicolumn{1}{c}{ NPA } & Participant & \multicolumn{1}{c}{ NPA } \\
\hline AGC & $(4: 14) 100=28.57 \%$ & JIS & $(7: 14) 100=50.00 \%$ \\
DCF & $(14: 15) 100=93.33 \%$ & AFM & $(7: 14) 100=50.00 \%$ \\
APC & $(6: 15) 100=40.00 \%$ & FMS & $(12: 15) 100=80.00 \%$ \\
BFG & $(14: 15) 100=93.33 \%$ & SAA & $(6: 15) 100=40.00 \%$ \\
ACR & $(10: 15) 100=66.67 \%$ & JARC & $(3: 14) 100=21.43 \%$ \\
MBF & $(14: 15) 100=93.33 \%$ & JORC & $(11: 15) 100=73.33 \%$ \\
IGL & $(7: 15) 100=46.67 \%$ & DMF & $(13: 15) 100=86.67 \%$ \\
DLC & $(3: 15) 100=20.00 \%$ & PGM & $(6: 14) 100=42.86 \%$ \\
DGM & $(6: 14) 100=42.86 \%$ & CMR & $(14: 15) 100=93.33 \%$ \\
VGC & $(6: 14) 100=42.86 \%$ & AFB & $(5: 14) 100=35.71 \%$ \\
\hline
\end{tabular}

\section{Eye gaze}

Forty percent of the participants exhibited expected eye contact with the interviewer during the interactions (item 1). Nonetheless, the tendency that prevailed in the rest of the cases $(60 \%)$ was avoidance of visual contact. A similar percentage (35\%) displayed a natural type of gaze. This was not the case in most of the subjects diagnosed with AS: 65\% avoided direct eye contact (item 2), looking at other places in the room where the communication exchange was taking place.

Therefore, the nonverbal dimension of eye gaze proved altered in most participants with AS. These deficits were related to an indirect and monotonous gaze that was seldom directed toward the interlocutor's face. It is easy to associate this conduct with a patient's lack of interest and receptivity. However, this perception rarely coincides with this type of symptomatic gaze present in subjects with ASD.

\section{Facial expression}

Once again, $40 \%$ of participants did not show any deficit related to the type of facial expression (item 3). Most commonly, facial expressions were insufficient or were not activated when the linguistic message taking place required it. These alterations suggest a low repertory of facial expressions in people with AS, who, on many occasions, produce a sensation of flatness during exchanges. Less frequently, but in some cases, facial expression did not compensate the linguistic content of verbal emissions but pretended to substitute it. When this happened, the participant tended to force facial expression, and this attempt - always used to express emotions - was artificial and deliberated.

A somewhat higher percentage (50\%) managed to adapt facial expressions to the linguistic content of emissions, and these were coherent with the context 
they originated in, as well as the emotional state of the evaluated subject (item 4). Nonetheless, this nonverbal communication item is closely related to the previous item. In short, facial expressivity - or, more precisely, facial flatness - is symptomatic of a nonverbal communication deficit shared by most participants with AS.

\section{Body language and posture}

Forty-five percent of the participants maintained appropriate body posture during verbal interactions (item 5). The predominant position was rigid and tense (30\%), even though there was a certain equivalency with cases in which the participant manifested hyperkinetic and restless behavior (25\%).

In any case, the appropriateness of posture is an affected aspect in individuals diagnosed with ASD. An extremely rigid posture can be symptomatic, for example, of anxiety; if a stable position is not kept, it shows lack of interest.

\section{Proxemics}

Unlike the previous dimensions, $95 \%$ of participants with AS adequately regulated physical contact with the interlocutor, and the distances between interviewer and patient were also the expected ones (item 6). All in all, it should be emphasized that proxemic demands required by the context in which the interactions took place did not show all the possible deterioration in nonverbal communication that could be manifested in other daily situations.

There are many authors that consider the existence of important proxemic deficits in communication behavior of people diagnosed with AS; that is, these individuals have difficulties when it comes to judging socially appropriate distances that are also necessary for effective interaction (Attwood, 2001; Wing, 2001; Jones, 2004; Rodríguez-Muñoz \& Ridao, 2011). More specifically, authors like Myles, Barnhill and Hagiwara (2001) conclude that there is a lack of understanding of interpersonal space in people with AS, who sometimes maintain extreme physical contact with strangers or touch them as if they were objects.

\section{Gestures}

Only $35 \%$ of the participants produced gestures naturally during communication exchanges (item 7). Therefore, most of them accompanied verbal expression with deliberate (not spontaneous) gestures. In some cases, gestures superseded the verbal message that was meant to be transmitted.

The percentage of individuals that carried out the expected amount of gestures was even lower, $25 \%$ (item 8 ). In general, gestural capacity was very limited; $50 \%$ of the individuals developed a reduced gestural repertory, while $25 \%$ were characterized by excessive gestures.

Forty-five percent of the evaluated subjects adjusted their gestures to the content of the verbal messages as well as to the context; in addition, under these circumstances, gestures where coherent with the emotional state they sought to 
communicate (item 9). In almost every case, this item is related to the previous one, since the absence of gestures prevents us from assessing their appropriateness correctly, and in this sense this item is not evaluated in $40 \%$ of the cases. Next, $15 \%$ of the participants presented gestures that were incoherent or disproportionate in relation to the content of the emissions.

Twenty-five percent added tics to their verbal expression (item 10). These involuntary movements were defined by impulsive blinking in both eyes on one occasion, and in a second occurrence by compulsive blinking of one eye only. Another subject pressed his lips automatically during conversation. Finally, in just one case the tics disorder had generalized and involuntary movements extended to different parts of the body.

\section{Paralanguage}

In $70 \%$ of the subjects that were assessed, the volume and tone of voice were sufficient for intelligibility (item 11). Among the participants with negative evaluations on this item, those who used a tone below average levels prevailed; it was less frequent to encounter excessively high voice tones through a whole interaction.

Somewhat lower (50\%) was the proportion of participants that adapted their elocution to rhythmic moderated guidelines, without producing sudden accelerations or decelerations (item 12). From a rhythmic point of view, people with AS frequently acquire some emphasis and do not complement it with many vocal inflections. This lack of sound shifts, just as useful for the transmission of attitudes and emotional states, gives place to monotony; this was an aspect evaluated negatively and was common in most of the assessed subjects.

Breathing was regulated correctly by $80 \%$ of the participants (item 13). However, sometimes we found ourselves with a faltering voice that is justified by the incapacity of some subjects when it comes to coordinating phonation and breathing, a decisive aspect for the adequate production of voice.

Seventy-five percent produced speech with no oral irregularities (item 14). The remaining portion of cases was distributed between articulatory difficulties (dyslalias), mainly rhotacism - a distorted pronunciation of the $[\mathrm{r}]$ sound, and subjects affected with dysphemia, who mostly tended to repeat the endings of words involuntarily. It is necessary to mention that no cases of echolalia were found.

Finally, most of the subjects with AS (80\%) did not turn to imitation of other voices during the interactions with the interviewer (item 15). Despite this, the remaining participants $(20 \%)$ changed the tone of their voice specifically when they introduced direct style into their answers.

\section{Conclusions and limitations of the study}

Nonverbal pragmatic disabilities in people with AS represent an important and under-studied research area that deserves more attention. In general, our results confirm the assumption that there are different alterations related to most of the 
nonverbal components into which the evaluation profile was divided. Mainly, these pathological manifestations referred to eye gaze, facial expressions, body language, posture and, above all, gestures.

However, some limitations of our study have to be noted, which demand further research efforts. First, the ideal score for neurotypicals is $100 \%$, but the real one would be placed in a lower range. Therefore, participants without AS should be involved in future studies in order to obtain a better interpretation of the observed deviations. Second, the QPPA and our nonverbal communication assessment profile score the items in a binary fashion, with either a positive or negative score for each item; however, a more fine-grained analysis would provide more accurate measurements. Finally, it is essential to extend the assessment of nonverbal pragmatic behaviors to more naturalistic contexts.

\section{Acknowledgments}

This paper stems from the $\mathrm{PhD}$ dissertation entitled Pragmatic deficits in the oral speech of children with Asperger syndrome, directed by Drs. Bañón-Hernández and Fornieles-Alcaraz, with International Mention and supported by FPU Research Grant AP2008-01707, funded by the Ministry of Education, Culture and Sport (Government of Spain). The research is part of the project "Ampliación y adaptación del corpus PERLA de datos clínicos en el marco del proyecto internacional Childes/Talkbank: perfiles pragmáticos y propuestas de intervención" (FFI2012-39325-C03-01), funded by the Ministry of Economy and Competitiveness (Government of Spain). It has been possible thanks to the admirable cooperation of the Asperger syndrome associations of Asturias and Valencia, to the participants in this study and their families.

\section{References}

American Psychiatric Association (2000). Diagnostic and statistical manual of mental disorders (IV Ed. Text Revision). Washington, DC: American Psychiatric Association.

Attwood, T. (2000). Strategies for improving the social integration of children with Asperger Syndrome. Autism, 4, 85-100.

Attwood, T. (2001). Asperger's syndrome: a guide for parents and professionals. London: Jessica Kingsley Publishers.

Attwood, T. (2006). The complete guide to Asperger's syndrome. London: Jessica Kingsley Publishers.

Baron-Cohen, S., Leslie, A.M., \& Frith, U. (1985). Does the autistic child have a 'theory of mind'? Cognition, 21, 37-46.

Bishop, D.V.M. (1998). Development of the Children's Communication Checklist (CCC): A method for assessing qualitative aspects of communicative 
impairment in children. The fournal of Child Psychology and Psychiatry, 39, 879-891.

Braverman, M., Fein, D., Lucci, D., \& Waterhouse, L. (1989). Affect comprehension in children with pervasive developmental disorders. Fournal of Autism and Developmental Disorders, 19, 301-316.

Cestero, A.M. (2006). La comunicación no verbal y el estudio de su incidencia en fenómenos discursivos como la ironía. Estudios de Lingüística. Universidad de Alicante, 20, 57-77.

Charman, T. \& Baird, G. (2002). Practitioner review: Diagnosis of autism spectrum disorder in 2- and 3-year-old children. Fournal of Child Psychology and Psychiatry, 43, 289-305.

Clapham, C. (2013). Principles of assessment. Subject Centre for Languages, Linguistics and Area Studies Good Practice Guide. Retrieved 25.11.2013 from https://www.llas.ac.uk/resources/gpg/1398.

Dalton, K.M., Nacewicz, B.M., Johnstone, T., Schaefer, H.S., Gernsbacher, M.A., Goldsmith, H.H., Alexander, A.L., \& Davidson, R.J. (2005). Gaze fixation and the neural circuitry of face processing in autism. Nature Neuroscience, 8, 519-526.

Doody, J.P. \& Bull, P. (2011). Asperger's syndrome and the decoding of boredom, interest, and disagreement from body posture. Fournal of nonverbal behavior, $35,87-100$.

Ekman, P. (2004). ¿Qué dice ese gesto? Barcelona: RBA.

Gallardo, B. (2007). Pragmática para logopedas. Cádiz: Universidad de Cádiz.

Gallardo, B. (2009a). Valoración del componente pragmático a partir de datos orales. Revista de neurología, 48, S57-S61.

Gallardo, B. (2009b). Trastorno por déficit de atención y/o hiperactividad. Materiales $y$ analisis pragmático. Valencia: University-AVaLCC.

Gallardo, B. (2010). Habilidades lingüísticas pragmáticas en el síndrome de Williams. Revista de Investigación, 70, 71-93.

Gallardo, B. \& Moreno, V. (2005). Afasia no fluente. Materiales y análisis pragmático. Valencia: University-AVaLCC.

Gallardo, B. \& Moreno, V. (2011). Pragmática enunciativa en lesionados de hemisferio derecho: estudio contrastivo respecto a hablantes afásicos. Revista de Investigación Lingüística, 14, 23-34.

Gallardo, B. \& Sanmartín, J. (2005). Afasia fluente. Materiales para su estudio. Valencia: University-AVaLCC.

Gallardo, B., Moreno, V., \& Pablo M.R. (2011). Lesionados de Hemisferio Derecho. Materiales y análisis pragmático. Valencia: University-AVaLCC.

Garayzábal, E. (2005). Síndrome de Williams. Materiales y análisis pragmático. Valencia: University-AVaLCC.

Gillberg, I.C. \& Gillberg, C. (1989). Asperger syndrome - some epidemiological considerations: A research note. Fournal of Child Psychology and Psychiatry, 30, 631-638. 
Grossman, J.B., Klin, A., Carter, A.S., \& Volkmar, F.R. (2000). Verbal bias in recognition of facial emotions in children with Asperger's syndrome. fournal of Child Psychology, and Psychiatry, 41, 369-379.

Hall, E.T. (1963). A system for the notation of proxemic behavior. American Anthropologist, 65, 1003-1026.

Hernández, N., Metzger, A., Magne, R., Bonnet-Brilhault, F., Roux, S., Barthelemy, C., \& Martineau, J. (2009). Exploration of core features of a human face by healthy and autistic adults analyzed by visual scanning. Neuropsychologia, 47, 1004-1012.

Hernández, C., Serra, E., \& Veyrat, M. (2007). Afasia: casos mixtos. Materiales y análisis lingüístico. Valencia: University-AVaLCC.

Jones, R.J. (2004). SAA RED: A Burkean pentadic model to assist those having Asperger's syndrome with interpersonal communication. Texas: Texas Tech University Electronic Theses and Dissertations. Retrieved 30.07.2012 from http://repositories.tdl.org/ttu-ir/handle/2346/14455.

Kendon, A. (1984). Did gesture escape the curse of Babel? In A. Wolfgang (Ed.), Nonverbal Behavior. Perspectives, Applications, Intercultural Insights (pp. 75114). Lewiston, CJ: Hogrefe.

Klin, A., Jones, W., Schultz, R., Volkmar, F., \& Cohen, D. (2002). Visual fixation patterns during viewing of naturalistic social situations as predictors of social competence in individuals with autism. Archives of General Psychiatry, 59, 809-816.

Knapp, M.L. (1992). La comunicación no verbal. El cuerpo y el entorno. Barcelona: Paidós.

McGovern, C.W. \& Sigman, M. (2005). Continuity and change from early childhood to adolescence in autism. Journal of Child Psychology and Psychiatry, 46, 401-408.

Mehrabian, A. (1972). Nonverbal communication. Chicago: Adeline-Atherton.

Myles, B.S., Barnhill, G.P., \& Hagiwara, T. (2001). A synthesis of studies on the intellectual, academic, social/emotional and sensory characteristics of children and youth with Asperger syndrome. Education and Training in Mental Retardation and Developmental Disabilities, 36, 1-10.

Nascimiento, N. (2005). Comunicación no verbal: Algunas diferencias gestuales entre España y Brasil. Lingüística en la Red, 2, 1-14.

Neumann, D., Spezio, M.L., Piven, J., \& Adolphs, R. (2006). Looking you in the mouth: Abnormal gaze in autism resulting from impaired top-down modulation of visual attention. Social Cognitive and Affective Neuroscience, 1, 194-202.

Niemi, J., Otsa, L., Evtyukova, A., Lehtoaro, L., \& Niemi, J. (2010). Linguistic reflections of social engagement in Asperger discourse and narratives: A quantitative analysis of two cases. Clinical Linguistics \& Phonetics, 24, 928-940.

Poyatos, F. (1977). The morphological and functional approach to kinesics in the context of interaction and culture. Semiótica, 20, 197-228. 
Poyatos, F. (1994). La comunicación no verbal I: Cultura, lenguaje y conversación. Madrid: Istmo.

Prutting, C.A. \& Kittchner, D.M. (1987). A clinical appraisal of the pragmatic aspects of language. Journal of Speech and Language Disorders, 52, 105-119.

Reed, C.L., Beall, P.M., Stone, V.E., Kopelioff, L., Pulham, D.J., \& Hepburn, S.L. (2007). Brief report: Perception of body posture - what individuals with autism spectrum disorder might be missing. Fournal of Autism and Developmental Disorders, 37, 1576-1584.

Ringman, J.M. \& Jankovic, J. (2000). Occurrence of tics in Asperger's syndrome and autistic disorder. Journal of Child Neurology, 15, 394-400.

Rodríguez-Muñoz, F.J. (2009). Síndrome de Asperger. Materiales y aproximación pragmalingüística. Valencia: University-AVaLCC.

Rodríguez-Muñoz, F.J. (2011). La comunicación no verbal en pacientes con síndrome de Asperger: Una experiencia de enseñanza virtual. Lenguaje y Textos, 34, 91-98.

Rodríguez-Muñoz, F.J. (2012). La conciencia pragmática de adultos con síndrome de Asperger. Revista de Logopedia, Foniatría y Audiología, 32, 21-31.

Rodríguez-Muñoz, F.J. (2013). Evaluación pragmática de niños con síndrome de Asperger. Munich: Lincom Verlag.

Rodríguez-Muñoz, F.J. \& Ridao Rodrigo, S. (2011). La docencia virtual en el tratamiento comunicativo de enfermedades raras: Descripción de un curso destinado a profesionales en síndrome de Asperger. RED-U. Docencia Universitaria en la Sociedad del Conocimiento, 3, 1-16.

Roth, F.P. \& Spekman, N.J. (1984). Assessing the pragmatic abilities of children: Part 1. Organizational framework and assessment parameters. Journal of Speech and Hearing Disorders. 49, 2-11.

Rutter, M., Bailey, A., \& Lord, C. (2003). The Social Communication Questionnaire. Los Angeles, CA: Western Psychological Services.

Shattuck, P.T., Seltzer, M.M., Greenberg, J.S., Orsmond, G.I., Bolt, D., Kring, S., Lounds, J., \& Lord, C. (2007). Changes in autism symptoms and maladaptive behaviors in adolescents and adults with an autism spectrum disorder. fournal of Autism and Developmental Disorders, 37, 1735-1747.

Sigman, M. \& Capps, L. (2000). Niños y niñas autistas: Una perspectiva evolutiva. Madrid: Ediciones Morata.

Szatmari, P., Brenner, R., \& Nagy, J. (1989). Asperger's syndrome: A review of clinical features. Canadian Journal of Psychiatry, 34, 554-560.

Tantam, D. \& Girgis, S. (2009). Recognition and treatment of Asperger syndrome in the community. British Medical Bulletin, 89, 41-62.

Wing, L. (2001). The autistic spectrum, a parents' guide to understanding and helping your child. Berkeley, CA: Ulysses Press.

World Health Organization (1992). The ICD-10 classification of mental and behavioural disorders: Clinical descriptions and diagnostic guidelines. Geneva, Switzerland: World Health Organization. 